\title{
Low Frequency Radio Emission of Pulsar PSR J1907+0919 Associated with the Magnetar SGR 1900+14
}

\author{
Yu. P. Shitov, V. D. Pugachev and S. M. Kutuzov \\ Pushchino Radioastronomy Observatory, Astro Space Center of Lebedev \\ Physical Institute, Pushchino, 142292, Russia, shitov@prao.psn.ru
}

\begin{abstract}
The soft gamma repeater SGR 1900+14 was observed in Pushchino observatory since 1988 December using BSA radio telescope operating at $111 \mathrm{MHz}$. We have detected the pulsed radio emission (Shitov 1999) with the same $5.16 \mathrm{~s}$ period that was reported earlier for this object (Hurley et al. 1998). The timing analysis has shown that this new radio pulsar PSR J1907+0919 associated with SGR $1900+14$ has a superstrong magnetic field, which is $8.1 \cdot 10^{14} \mathrm{G}$, thereby confirming that it is a "magnetar" (Duncan \& Thompson 1992; Kouveliotou et al. 1999). The dispersion measure of PSR J1907+0919 is $281.4(9) p c \cdot \mathrm{cm}^{-3}$ which gives an estimate of the pulsar's distance as about $5.8 \mathrm{kpc}$.
\end{abstract}

\section{Introduction}

Among known soft gamma- ray repeaters to date, only SGR $1900+14$ and SGR $1806-20$ are the objects for which a secular spin-down of the pulse periods $(5.16$ s and $7.47 \mathrm{~s}$ accordingly) with $\dot{P}$ of order $10^{-10} \mathrm{~s} / \mathrm{s}$ was detected and thereby was established that these SGRs are neutron stars with a superstrong magnetic field of order $10^{15} \mathrm{G}$ (Kouveliotou et al. 1998; Kouveliotou et al. 1999), called as a "magnetars" (Duncan \& Thompson 1992).

Since the end of 1998 we carried out the observations of the SGR $1900+14$ at low frequency $(111 \mathrm{MHz})$ and have detected the periodic pulsed radio emission from this magnetar (Shitov 1999). In this paper we report the results of our observations obtained till August 1999.

\section{Observations}

The observations of the magnetar SGR $1900+14$ were started since 1998 December using the BSA radio telescope operating now at the new frequency of 111 $\mathrm{MHz}$. (In 1998 October BSA - wavelength dipole array with dimensions of 187 x $384 \mathrm{~m}$, transit time of $200 \mathrm{~s} / \cos (\delta)$ - was reconstructed to shift the previous operating frequency of $102.5 \mathrm{MHz}$ to the new one of $111 \mathrm{MHz}$ (Kutuzov et al. 1999) ) We used $128 \times 20 \mathrm{kHz}$ filterbank receiver and multichannel recording system to record the individual pulses during the BSA transit time. Different sampling intervals of 22.6816 and $20.1728 \mathrm{~ms}$ were used. All further processing procedures: cleaning of the noise signal in a each frequency channel, dedisper- 
SGR $1900+14$

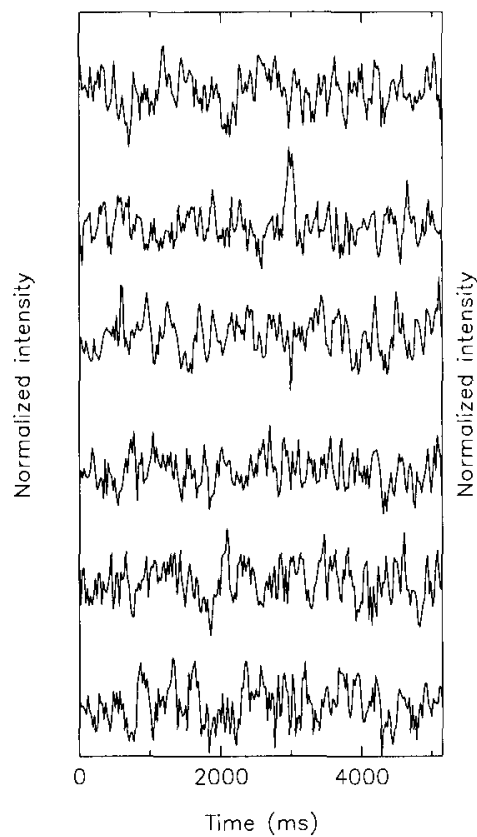

SGR $1900+14$

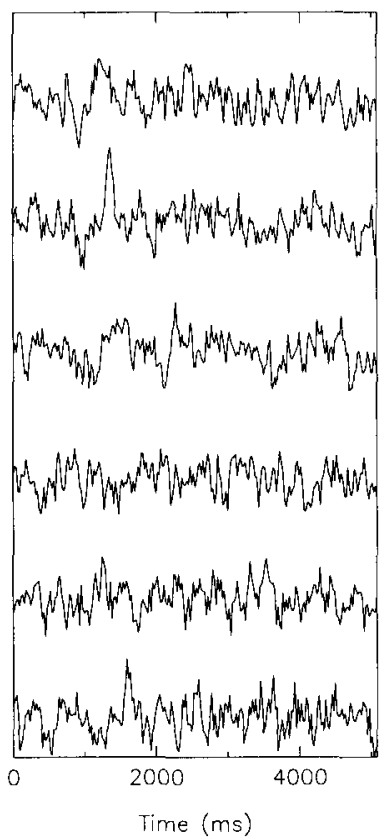

Figure 1. The dedispersed with different DM values and folded with the $5.161 \mathrm{~s}$ period records of SGR $1900+14$, obtained at $111 \mathrm{MHz}$ in 1998 December 12 (left) and 1999 January 6 (right). DM $=0$ (bottom), $70,140,210,281,350$

sion, spectral analysis, the folding of dedispersed data for integrated pulse profile and the timing analysis were made "off line".

\section{The detection of radio pulses from SGR $1900+14$}

For the first time we have detected pulsed with 5.161-s period radio emission from SGR $1900+14$ in 1998 December 12, when as result of dedispersion procedures the integrated pulse profile with a good enough signal/noise ratio appeared with a dispersion measure (DM) of about $280 \mathrm{pc} \cdot \mathrm{cm}^{-3}$. It was surprising that the revealed profile was quite narrow, with the width of about 7.5 . The next radio detection of SGR 1900+14 like the first one was only in 1999 January 6 (note that between this dates the antenna array was often covered by hoar-frost). Fig. 1 demonstrates these two detection of the SGR signals at $111 \mathrm{MHz}$.

During subsequent regular observations the pulses from SGR $1900+14$ with the same DM values and similar pulse width of about $100 \mathrm{~ms}$ were detected repeatedly. The estimated for the best records mean flux density at $111 \mathrm{MHz}$ is approximately $50 \mathrm{mJy}$. The measured value of $D M=281.4(9) \mathrm{pc} \cdot \mathrm{cm}^{-3}$. So far as the period derivative $\dot{P}$ was known for the magnetar with the limited 
SGR $1900+14$

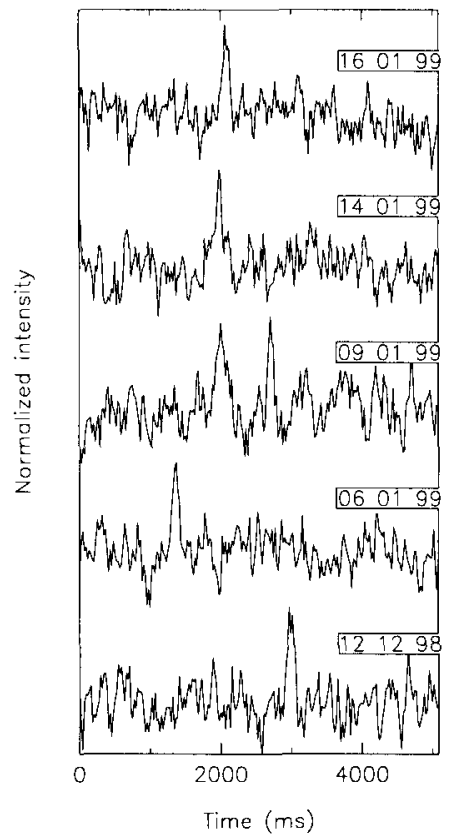

SGR $1900+14$

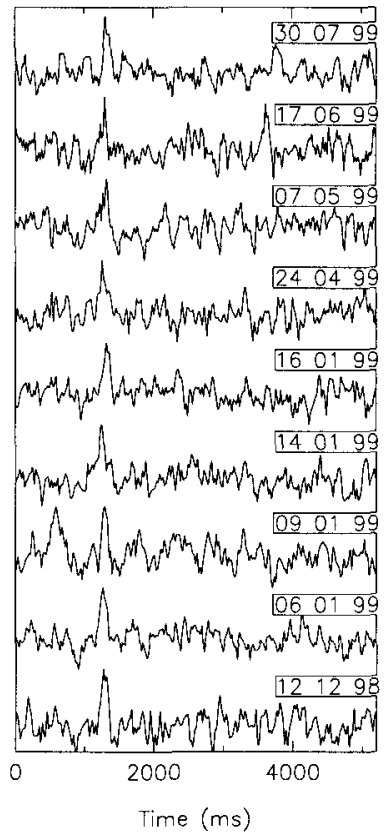

Figure 2. Left - some of the first records of SGR $1900+14$ obtained at $111 \mathrm{MHz}$. After improving of the $P$ and $\dot{P}$ values the pulse profiles on January 14 and 16 were observed in the precalculated phases. Right - the time aligned pulse profiles of SGR $1900+14$ barycentric phases of which are in accordance with the best fitted $P, \dot{P}$, and $\ddot{P}$ values.

accuracy the pulses were recorded in a unpredictable phases (Fig.2, left) till the middle of January 1999 when $P$ and $\dot{P}$ were improved as a result of the timing analysis. Fig.2 (right) demonstrates some pulse profiles of SGR $1900+14$, the phase of which aligned in an accordance with the timing solution.

There were a number of records in which we have detected interpulses with amplitude (and, of course, dispersion measure) like the main pulse, and which were located in different phases. Two examples of these interpulses are seen in Fig. 2 (0901 99 and 170699 ).

\section{Timing analysis}

For timing analysis of our data as the initial parameters of SGR $1900+14$ we used the values of $\mathrm{P}=5.160199(2) \mathrm{s}, \dot{P}=1.14(23) \cdot 10^{-10} \mathrm{~s} / \mathrm{s}, \mathrm{MJD}=51056.0$, obtained by Kouveliotou et al. (1999) in 1998 August 28. The exact position of the magnetar: R.A. $(J 2000)=19 h 07 m 14.33$, Dec. $(J 2000)=09^{\circ} 19^{\prime} 20^{\prime \prime} .1$, obtained with the VLA observations of an outburst of relativistic particles from SGR $1900+14$ (Frail, Kulkarni \& Bloom 1999) we used in our analysis. The first 


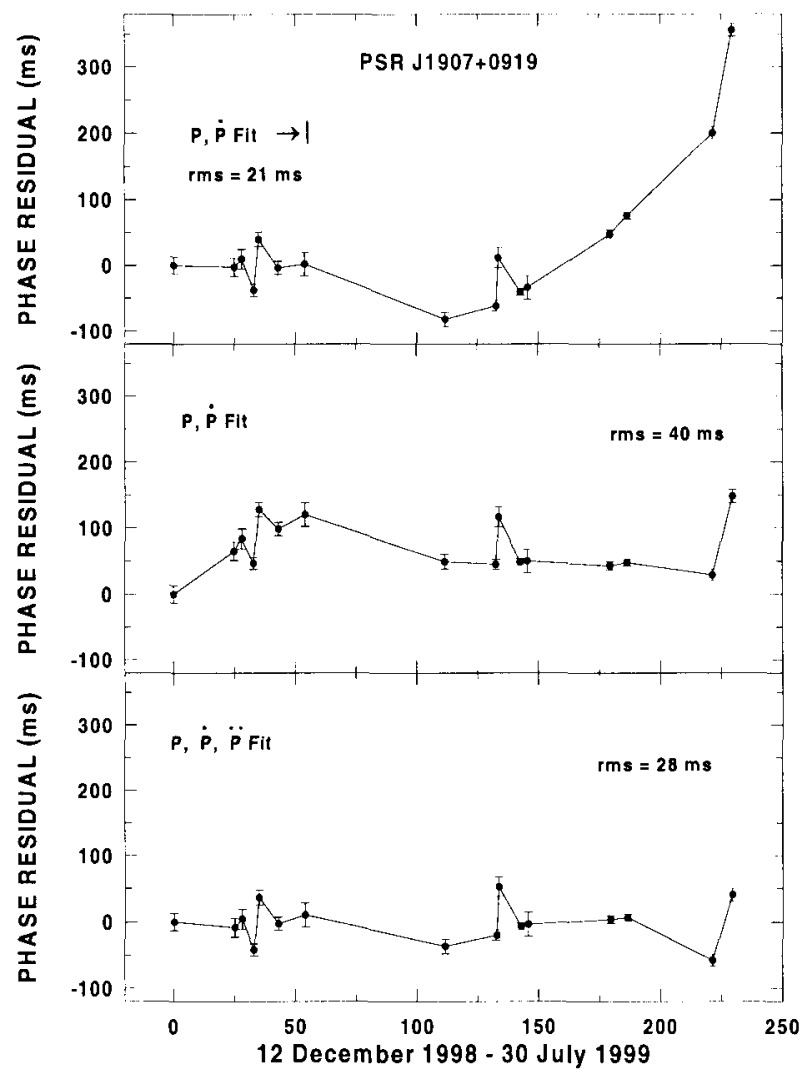

Figure 3. Timing residuals for different parameters $P, \dot{P}$ and $\ddot{P}$ : from IAUC 7110 (top); $\ddot{P}=0.0$ (middle); best fitted (bottom)

timing solution for $P$ and $\dot{P}$ was found for the first records, obtained in the date interval Dec. 121998 - Jan. 14 1999, after that in the subsequent observations with improved parameters the pulses were detected in the precalculated phases. In IAUC 7110 we have reported the solution: $\mathrm{P}=5.161297854(83) \mathrm{s}, \dot{P}=$ $1.23228(34) \mathrm{s} / \mathrm{s}$, which was found for the interval Dec. 121998 - Feb. 41999.

In this paper we present the results, obtained during Dec. 121998 - July 301999 . We have selected 16 best records and found for this dates interval the following timing solution: $\mathrm{P}=5.161297899(67) \mathrm{s}, \dot{P}=1.23197(15) \cdot 10^{-10} \mathrm{~s} / \mathrm{s}$, $\ddot{P}=+0.53(14) \cdot 10^{-20} \mathrm{~s} / \mathrm{s} / \mathrm{s}, \mathrm{MJD}=51159.4605$ with the RMS of phase residual of $28 \mathrm{~ms}$. The inclusion of the second period derivative essentially improved the timing in this interval of dates (see Fig. 3). For the epoch MJD $=51056.0$, found parameters give $\mathrm{P}=5.1601969 \mathrm{~s}$, that is in a good agreement with Kouveliotou's et al. (1999) measurements. 


\section{Conclusions}

New radio pulsar PSR J1907+0919 associated with the soft gamma repeater SGR $1900+14$ is representative of a new class of pulsars with a superstrong magnetic field, slow down value of which for this pulsar is $8.1 \cdot 10^{14} \mathrm{G}$. Presented results confirm that this object is a magnetar (Duncan \& Thompson 1992; Kouveliotou et al. 1999). The pulsar distance of about $5.8 \mathrm{kpc}$ determined from dispersion measure $D M=281 p c \cdot \mathrm{cm}^{-3}$ supports the suggested earlier in a number of papers genetic connection of SGR $1900+14$ with supernova remnants SNR G42.8+0.6. As timing analysis have shown there is no evidence for binary or bital motion of this pulsar, at least with $P_{\text {orb }}<250$ days and with $a \cdot \sin i>60$ ms.

Acknowledgments. This work was supported partly by grant INTAS 96 0154 .

\section{References}

Duncan, R. C., \& Thompson, C. 1992, ApJ, 392, L9

Frail, D.A., Kulkarni, S.R. \& Bloom, J.S. 1999, Nature, 398, 127

Hurley, K., Kouveliotou, C., Murakami, T., \& Strohmayer, T. 1998, IAU Circ. 7001

Kouveliotou, C., et al. 1998, Nature, 393, 235

Kouveliotou, C., Strohmayer, T., Hurley, K., van Paradijs, J., Finger, M. H., Dieters, S., Woods, P., Thompson, C. \& Duncan, R. C. 1999, ApJ, 510, L115

Kutuzov, S. M., et al. 1999 in Lebedev Physical Institute Proc., submitted Shitov, Yu.P. 1999, IAU Circ. 7110 